\title{
Variceal hemorrhage of the colon secondary to pancreatic cancer
}

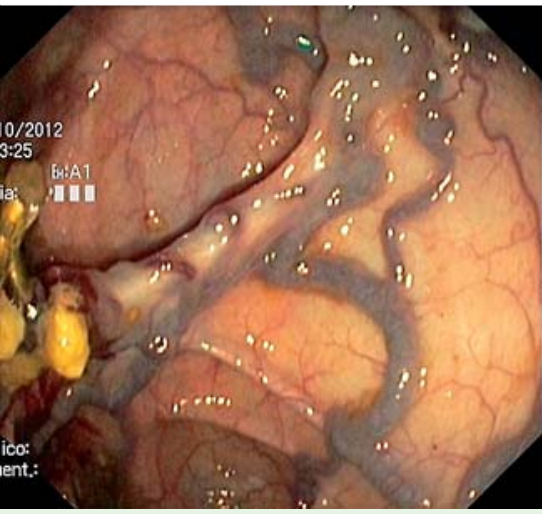

Fig. 1 Endoscopic view of colonic lumen in a 71-year-old woman with previous segmental colonic resection for adenocarcinoma, who presented with hematochezia. Colonoscopy showed colon varices extending proximal to the anastomosis, with superficial venules (red wale markings).

A 71-year-old woman was admitted due to recurrent lower gastrointestinal bleeding. She had undergone segmental colonic resection and adjuvant chemotherapy for colon adenocarcinoma 10 years earlier, without recurrence on follow-up. She reported experiencing several self-limited episodes of hematochezia over the past 6 months, without other symptoms.

Blood tests showed iron deficiency anemia (hemoglobin $9.9 \mathrm{~g} / \mathrm{dL}$ ). Colonoscopy revealed dilated, tortuous, bluish vessels protruding into the lumen and extending proximal to the anastomosis, numerous superficial venules, and fresh blood and clots in the lumen ( $\bullet$ Fig. 1 ). Hemostasis was achieved by adrenaline injection and hemostatic clips. Computed tomography angiography demonstrated prominent collateral vessels near the colonic anastomosis, without signs of thrombosis ( Fig. 2). In addition, a nodular density adjacent to the pancreatic uncinate process was noted, with superior mesenteric vessels involvement. Subsequent endoscopic ultrasound (EUS) showed a 31-mm hypoechoic pancreatic head mass ( Fig.3), with invasion of the splenoportal confluence, generating a "stop" image on Doppler ultrasound ( $\bullet$ Fig.4). Transbulbar EUS-guided fine-needle aspiration (22-gauge needle) was performed. Pathologic analysis revealed pan-

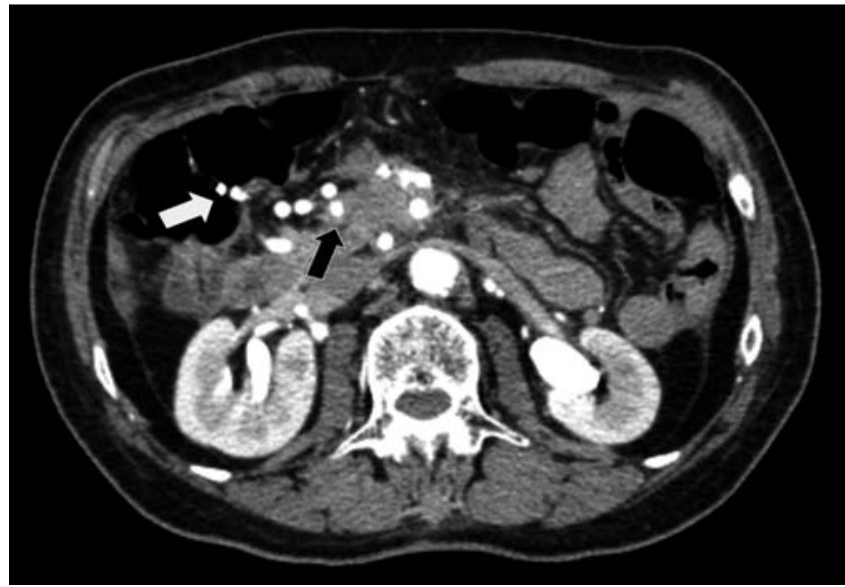

Fig. 2 Computed tomography image obtained after the administration of intravenous contrast showing prominent collateral vessels adjacent to the colonic anastomosis (white arrow), without signs of thrombosis. In addition, a nodular density adjacent to the pancreatic uncinate process was observed (black arrow).

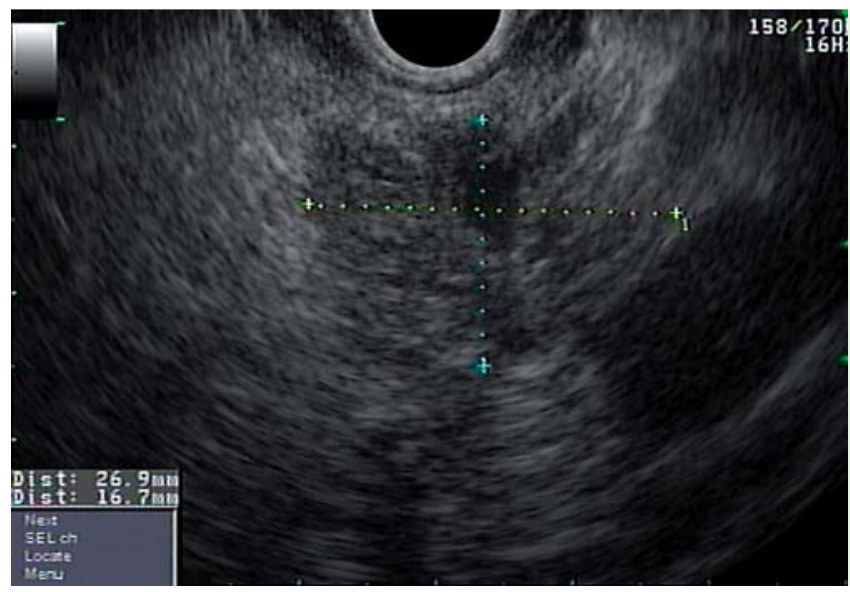

Fig. 3 Endoscopic ultrasound image showing a hypoechoic pancreatic head mass, with $31 \mathrm{~mm}$ dimension and irregular margins.

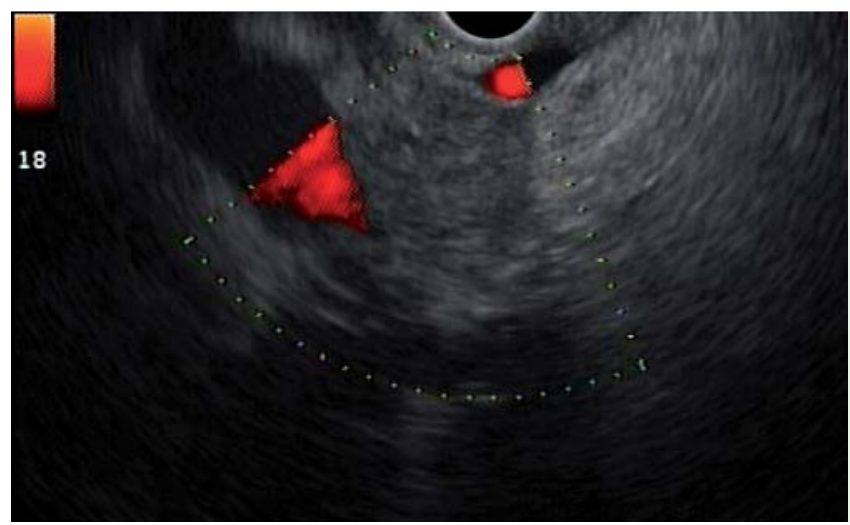

Fig. 4 Endoscopic Doppler ultrasound image demonstrating invasion of the splenoportal confluence by the pancreatic mass, which generates a "stop" image on Doppler sign.

creatic ductal adenocarcinoma ( $\bullet$ Fig.5). Due to recurrent colonic bleeding, a vascular stent was placed through percutaneous transhepatic selective portography (॰ Fig. 6).

Colonic varices are a very rare cause of lower gastrointestinal bleeding, with a reported incidence of $0.07 \%$ [1]. Portal hy- pertension is the most common etiology. Uncommon causes are congestive heart failure, mesenteric vein thrombosis, pancreatitis with splenic vein thrombosis, adhesions and, rarely, mesenteric vein obstruction [2]. It should prompt thorough evaluation, but can be idiopathic [1-3]. The present case is a peculiar con- 

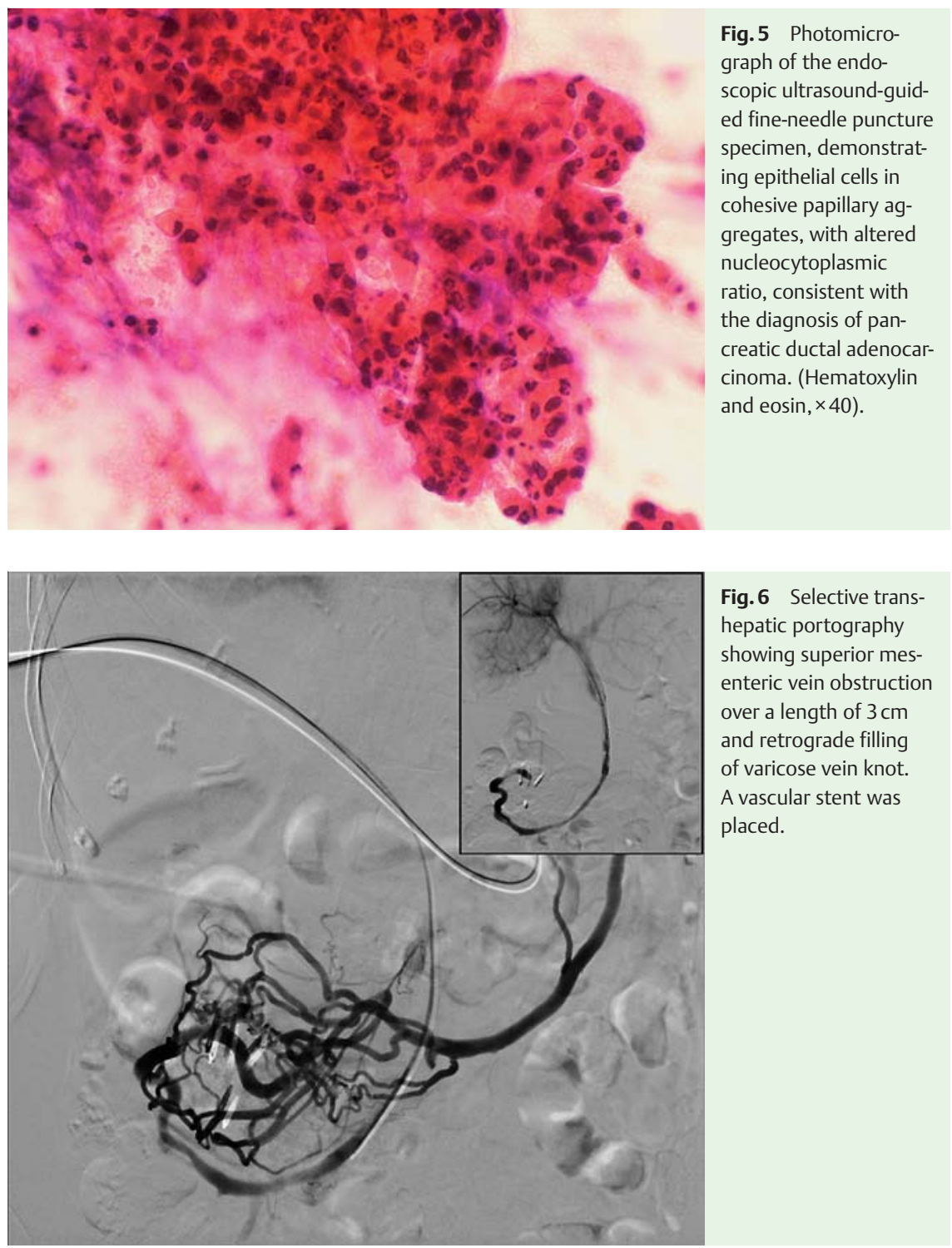

dition - a patient with previous segmental colectomy due to carcinoma, presenting with recurrent hematochezia as a result of variceal hemorrhage due to a second primary (pancreatic) carcinoma and
Endoscopy_UCTN_Code_CCL_1AD_2AF

\section{Competing interests: None}

\section{Teresa Pinto-Pais ${ }^{1}$, Sónia Sousa Fernandes ${ }^{1}$, Luísa Proença ${ }^{1}$, Pedro Roquete ${ }^{2}$, Tiago Pereira ${ }^{3}$, João Carvalho' ${ }^{1}$, José Fraga ${ }^{1}$}

${ }^{1}$ Department of Gastroenterology and Hepatology, Centro Hospitalar de Gaia/ Espinho, Vila Nova de Gaia, Portugal

2 Department of Histopatology, Laboratório Rodrigues Pereira, Porto, Portugal

${ }^{3}$ Department of Radiology, Centro Hospitalar de Gaia/Espinho, Vila Nova de Gaia, Portugal

Fig. 6 Selective transhepatic portography showing superior mesenteric vein obstruction over a length of $3 \mathrm{~cm}$ and retrograde filling of varicose vein knot. A vascular stent was placed.

mesenteric obstruction. This case highlights the importance of considering colonic varices in the differential diagnosis of lower gastrointestinal bleeding and the importance of thorough investigation.

\section{References}

1 Han JH, Jeon WJ, Chae HB et al. A case of idiopathic colonic varices: a rare cause of hematochezia misconceived as tumor. World J Gastroenterol 2006; 12: 2629-2632

2 Sohn W, Lee HL, Lee KN. Variceal hemorrhage of ascending colon. Clin Gastroenterol Hepatol 2012; 10: A24

3 Francois F, Tadros C, Diehl D. Pan-colonic varices and idiopathic portal hypertension. J Gastrointestin Liver Dis 2007; 16: $325-$ 328

Bibliography

DOI http://dx.doi.org/

10.1055/s-0034-1377763

Endoscopy 2014; 46: E500-E501

(c) Georg Thieme Verlag KG

Stuttgart · New York

ISSN 0013-726X

Corresponding author

Teresa Pinto-Pais, MD

Department of Gastroenterology and Hepatology

Centro Hospitalar de Gaia/Espinho

Rua Conceicao Fernandes

Vila Nova de Gaia 4434-502

Portugal

Fax: $+351-227865100$

teresapintopais@gmail.com 